
allemande

46-1 | 2014

Les fondements normatifs de l'État constitutionnel moderne en Allemagne. Une approche pluridisciplinaire

\title{
Le rôle de la pensée de Gustav Radbruch dans la refondation de l'État de droit démocratique après 1945
}

Nathalie Le Bouëdec

\section{OpenEdition}

Journals

Édition électronique

URL : https://journals.openedition.org/allemagne/1276

DOI : 10.4000/allemagne. 1276

ISSN : 2605-7913

Éditeur

Société d'études allemandes

Édition imprimée

Date de publication : 30 juin 2014

Pagination : 83-94

ISSN : 0035-0974

Référence électronique

Nathalie Le Bouëdec, «Le rôle de la pensée de Gustav Radbruch dans la refondation de l'État de droit démocratique après 1945 », Revue d'Allemagne et des pays de langue allemande [En ligne], 46-1 | 2014, mis en ligne le 29 juillet 2019, consulté le 18 mai 2021. URL : http://journals.openedition.org/ allemagne/1276; DOI : https://doi.org/10.4000/allemagne.1276 


\section{Le rôle de la pensée de Gustav Radbruch dans la refondation de l'État de droit démocratique après 1945}

\section{- Nathalie Le Bouëdec*}

Dans le cadre d'une réflexion sur les fondements normatifs de l'État constitutionnel allemand, la période qui entoure la création de la République fédérale présente incontestablement un intérêt particulier. Il s'agissait en effet de reconstruire un État de droit démocratique sur les ruines de l'État totalitaire et également, il ne faut pas l'oublier, sur celles de la première démocratie allemande, celle de Weimar - toute la question étant de savoir sur quelles bases, quels fondements. Cette question conduit à s'intéresser aux filiations, héritages et réappropriations qui ont pu être à l'œuvre dans ce processus. C'est dans cette perspective que nous voulons analyser ici le rôle qu'a pu jouer la pensée d'un juriste comme Gustav Radbruch qui, au vu de son engagement républicain sous Weimar et de sa non compromission avec le régime nazi, pouvait $a$ priori servir de point d'ancrage pour une telle refondation. Si le très fort retentissement de ses écrits de l'après-guerre et notamment de la fameuse «formule Radbruch » ( Radbruchsche Formel ») semble aller dans ce sens, la réception de sa pensée s'avère en réalité à maints égards paradoxale. Ces paradoxes apparaissent lorsque l'on étudie deux champs de discours et de réflexion clés de cette période: la reconstruction de l'État de droit, d'une part, celle de la démocratie, d'autre part.

En guise de préambule, il n'est pas inutile de rappeler très brièvement certaines données concernant Radbruch: né en 1878, professeur de droit spécialisé en droit pénal et philosophie du droit, il se distingua sous Weimar par son engagement républicain et social-démocrate - il fut notamment député et ministre de la Justice. Après avoir fait partie des premiers universitaires déchus de leur poste en 1933, Radbruch ne put retrouver ses fonctions qu'en 1945 avant de décéder en 1949, quelques mois seulement après la création de la République fédérale d'Allemagne. Sa notoriété repose pour beaucoup, ce qui constitue un premier paradoxe si l'on considère la durée relative de

\footnotetext{
* Maître de conférences en études germaniques, Université de Bourgogne.
} 
chaque période, sur les écrits publiés au cours de ces quatre années et notamment sur l'essai « Injustice légale et droit supralégal » (« Gesetzliches Unrecht und übergesetzliches Recht») de 1946, considéré comme un des essais de philosophie du droit les plus influents du $\mathrm{xx}^{\mathrm{e}}$ siècle ${ }^{(1)}$.

Les fondements de sa philosophie du droit, influencée par le néokantisme, sont le dualisme méthodologique (Methodendualismus), autrement dit la distinction du devoir-être et de l'être et l'impossibilité de déduire l'un de l'autre, et le relativisme: les jugements de valeur (Werturteile), qui relèvent de la sphère du devoir-être, ne peuvent pas être objets de connaissance scientifique (Erkenntnis), mais seulement d'une profession de foi (Bekenntnis); on ne peut donc prouver la supériorité d'un système de valeurs sur un autre. Le droit est défini par Radbruch de façon non positiviste comme ce qui se rapporte à l'idée de droit (Rechtsidee), la réalité dont le sens est de servir l'idée de droit. Celle-ci, qui relève de la sphère de la valeur, englobe trois éléments: la justice (Gerechtigkeit), c'est-à-dire le principe d'égalité, le caractère adéquat du droit (Zweckmäßigkeit) par rapport à la conception du bien commun et la sécurité juridique (Rechtssicherheit). À l'intérieur de ce cadre, la pensée de Radbruch a connu des évolutions qui, on va le voir, ont leur importance dans le contexte de l'après-guerre.

\section{La refondation de l'État de droit : Radbruchsche Formel, positivisme et renaissance du droit naturel}

\section{Les écrits de l'après-guerre et la "formule Radbruch»}

Comme l'écrit Radbruch en ouverture de son essai « La rénovation du droit » en 1947, le droit, à l'image du pays tout entier, n'est plus qu'un champ de ruines. Aux juristes incombe la lourde tâche de déblayer le lieu de la destruction et d'y reconstruire l'édifice du droit ${ }^{(2)}$. «Déblayer le lieu de la destruction » (" die Stätte der Zerstörung aufzuräumen »), cela signifie bien sûr répondre au défi que représentaient pour la justice les crimes commis sous le nazisme. C'est à ce problème que s'attaque Radbruch dans son essai de 1946 «Injustice légale et droit supralégal».

Sa réflexion théorique part d'un enjeu pratique - il énumère ainsi plusieurs affaires pénales jugées en zone soviétique: la justice est sans cesse confrontée au problème de la validité des « lois » nazies, à la nécessité de juger des actes alors non punis, voire encouragés (comme les dénonciations), y compris ceux des juges ayant prononcé des sentences extrêmes sur la base de ces « lois ». Selon Radbruch, la validité d'une loi ne peut se fonder sur sa simple capacité à s'imposer. Toute loi positive présente certes l'intérêt de réaliser indépendamment de son contenu une valeur essentielle: la sécurité juridique. Mais cette dernière n'est pas la valeur décisive que le droit doit réaliser; cette valeur, c'est la justice.

1 Voir R. Dreier, S. L. Paulson, «Einführung in die Rechtsphilosophie Radbruchs », in: G. Radbruch, Rechtsphilosophie, Heidelberg, C.F. Müller, 1999, p. 245. Selon Horst Dreier, il s'agit même du passage le plus cité de toute la philosophie du droit du xx siècle ("Gustav Radbruch und die Mauerschützen », Juristenzeitung, 52 [1997], p. 423). Cet article témoigne justement du renouveau connu par la formule Radbruch après la réunification dans le cadre des procès dits des «tireurs du mur ", ces soldats estallemands ayant abattu des concitoyens tentant de fuir la RDA.

2 G. Radbruch, «Die Erneuerung des Rechts » (1946), in: Gustav Radbruch Gesamtausgabe (GRGA) 3: Rechtsphilosophie III, Heidelberg, C.F. Müller, 1990, p. 107. Sauf indication contraire, c'est nous qui traduisons. 
Les cas auxquels est confrontée la justice après 1945 illustrent en fait le conflit possible entre sécurité juridique et justice. Pour résoudre ce conflit, Radbruch propose la solution suivante, passée à la postérité sous le nom de «formule Radbruch » :

«Le conflit entre la justice et la sécurité juridique pourrait être résolu en posant que le droit positif, assuré par le pouvoir et par le fait d'être posé, prévaut quand bien même son contenu est injuste et inadéquat, à moins que la contradiction entre la loi positive et la justice n'atteigne un degré tellement insupportable que la loi, en tant que "droit non juste", doive céder devant la justice. Il est impossible de tracer une ligne de démarcation plus nette entre les cas d'injustice légale et les lois pourtant valides en dépit d'un contenu non juste; on peut toutefois procéder à une autre délimitation qui, elle, est tout à fait nette : là où la justice n'est même pas visée, où l'égalité, qui constitue le cœur de la justice, est consciemment niée lorsque le droit positif est posé, la loi n'est pas seulement du "droit non juste", mais elle est bien plutôt dénuée de toute nature juridique ${ }^{(3)}$.

Les cas d'" injustice légale » indiqués par Radbruch permettent de comprendre le contenu qu'il donne au concept de justice: il évoque des lois qui traitent des êtres humains comme des sous-hommes et sont une négation des droits de l'homme, comme les lois raciales de Nuremberg de 1935, ou la promulgation de peines très lourdes, telle la peine de mort, pour des faits de gravité très différente. Le « droit supralégal » correspond donc à un concept matériel de justice fondé sur les droits de l'homme, l'égalité et la dignité humaine - autrement dit, même si l'expression n'apparaît pas dans ce texte, à une forme de droit naturel. Le terme Naturrecht apparaît en revanche en bonne place dans l'essai de 1947 cité plus haut, où Radbruch exhorte à se souvenir de « la sagesse millénaire commune à l'Antiquité, au Moyen-Âge chrétien et à l'époque des Lumières, c'est-à-dire du fait qu'il existe un droit de rang supérieur à la loi, un droit naturel (Naturrecht), un droit divin (Gottesrecht), un droit rationnel (Vernunftrecht), en un mot, un droit supralégal à l'aune duquel l'injustice reste de l'injustice, même si elle est coulée dans le moule de la loi ${ }^{(4)}$. C'est à cette seule condition que les Allemands pourront se prémunir contre le retour d'un État fondé sur l'injustice (Unrechtsstaat).

\section{Fortune...}

La « formule Radbruch » a connu un retentissement exceptionnel. Les conditions de réception étaient indéniablement favorables: les Allemands avaient besoin de critères normatifs, mais aussi de concepts théoriques pour appréhender de façon critique et

3 «Der Konflikt zwischen der Gerechtigkeit und der Rechtssicherheit dürfte dahin zu lösen sein, daß das positive, durch Satzung und Macht gesicherte Recht auch dann den Vorrang hat, wenn es inhaltlich ungerecht und unzweckmäßig ist, es sei denn, daß der Widerspruch des positiven Gesetzes zur Gerechtigkeit ein so unerträgliches Maß erreicht, daß das Gesetz als "unrichtiges Recht” der Gerechtigkeit zu weichen hat. Es ist unmöglich, eine schärfere Linie zu ziehen zwischen den Fällen des gesetzlichen Unrechts und den trotz unrichtigen Inhalts dennoch geltenden Gesetzen, eine andere Grenzziehung aber kann mit aller Schärfe vorgenommen werden: wo Gerechtigkeit nicht einmal erstrebt wird, wo die Gleichheit, die den Kern der Gerechtigkeit ausmacht, bei der Setzung positiven Rechts bewußt verleugnet wurde, da ist das Gesetz nicht etwa nur "unrichtiges Recht", vielmehr entbehrt es überhaupt der Rechtsnatur. » G. RADBRUCH, "Gesetzliches Unrecht und übergesetzliches Recht » (1946), in: GRGA 3, p. 89. Traduction (modifiée): Michael Walz, Archives de philosophie du droit, 30 (1995) (Le procès), Paris, Sirey, p. 313 sq.

4 G. Radbruch, « Die Erneuerung des Rechts » (note 2), p. 108. 
rationnelle l'injustice extrême du Troisième Reich. La formule a ainsi permis d'articuler une protestation d'ordre moral et éthique ${ }^{(5)}$ qui s'est exprimée très tôt dans des jugements invoquant la justice et le droit naturel ${ }^{(6)}$. Son intérêt résidait également dans son orientation pratique : l'intention de Radbruch était bien de donner à la justice des instruments méthodologiques et terminologiques pour juger les crimes commis sous le nazisme. À cet égard, son argumentation allait tout à fait dans le sens de la législation alliée, et notamment des dispositions de la loi $\mathrm{n}^{\circ} 10 \mathrm{du}$ Conseil de contrôle $\mathrm{e}^{(7)}$ concernant les crimes contre l'humanité.

De nombreuses décisions de la justice de la RFA se sont par la suite appuyées sur la «formule Radbruch ». En juillet 1951, le Bundesgerichtshof rendit un jugement dans une affaire de dommages et intérêts réclamés à un ancien commandant du Volkssturm qui, en avril 1945, avait fusillé un déserteur et invoqué le « Katastrophenbefehl» de Himmler « autorisant " tout homme portant une arme à fusiller un déserteur sans aucune procédure. La cour jugea cet " ordre » dénué de tout caractère juridique en se fondant d'une part sur les principes du droit international définis au procès de Nuremberg, et d'autre part sur la formule Radbruch, avec une référence explicite à l'essai de $1946^{(8)}$. Six mois plus tard, il fut question, devant cette même cour, du sort de quatre anciens membres de la Gestapo ayant participé à la déportation de Juifs. Il s'agissait entre autre de déterminer si leurs actes étaient couverts par les décrets nationaux-socialistes. Une fois encore, le jugement conteste la validité de ces décrets en s'appuyant sur une argumentation et une terminologie très proches de celles de Radbruch, sans toutefois le citer: " des ordres qui ne visent même pas à la justice, nient consciemment l'idée d'égalité [... ] ne créent pas de droit " ${ }^{(9)}$. Son influence est moins nette dans les décisions de la Cour constitutionnelle (Bundesverfassungsgericht), mais s'y révèle durable. En 1968, celle-ci dut décider si un Juif ayant émigré aux Pays-Bas sous Hitler avait perdu la nationalité allemande sur la base d'un décret de 1941 (Ausbürgerungsverordnung

5 Voir dans ce sens H. Dreier, «Die Radbruchsche Formel. Erkenntnis oder Bekenntnis », in: H. MAyeR (éd.), Staatsrecht in Theorie und Praxis, Vienne, Manz, 1991, p. 128; "Gustav Radbruch und die Mauerschützen » (note 1), p. 423.

6 Le 13 novembre 1945, un tribunal de Wiesbaden avait ainsi jugé que les lois en vertu desquelles la propriété des Juifs avait été déclarée dévolue à l'État étaient en contradiction avec le droit naturel et nulles au moment de leur promulgation. Süddeutsche Juristenzeitung (SJZ), 1 (1946), p. 36.

7 Du 20 décembre 1946. Cf. J. Perels, « Die Restauration der Rechtslehre nach 1945 » (1984), in: ID., Demokratie und soziale Emanzipation, Hambourg, VSA, 1988, p. 38 sq. La loi stipulait entre autres que les crimes contre l'humanité ("Verbrechen gegen die Menschlichkeit») - le meurtre, l'extermination, l'asservissement, la déportation, la privation de liberté, la torture, le viol ou d'autres actes inhumains commis envers la population civile, de même que les persécutions pour des motifs politiques, raciaux ou religieux - constituaient des crimes aux yeux de la justice, et ce qu'il y ait eu ou non violation du droit national du pays où les actes avaient été commis.

8 Jugement du 12 juillet 1951, BGHZ, 3, p. 107: «Das Gesetz findet dort seine Grenze, wo es in Widerspruch zu den allgemein anerkannten Regeln des Völkerrechts oder zu dem Naturrecht tritt (OGHSt, 2, 271) oder der Widerspruch des positiven Gesetzes zur Gerechtigkeit ein so unerträgliches Maß erreicht, daß das Gesetz als "unrichtiges Recht” der Gerechtigkeit zu weichen hat. Wird der Grundsatz der Gleichheit bei der Setzung des positiven Rechts überhaupt verleugnet, dann entbehrt das Gesetz der Rechtsnatur und ist überhaupt kein Recht » (RADBRUCH, SJZ, 1946, 105 [107]).

9 «Anordnungen, die die Gerechtigkeit nicht einmal anstreben, den Gedanken der Gleichheit bewußt verleugnen [...], schaffen kein Recht». Jugement du 29 janvier 1952, BGHSt, 2, p. 238 sq. 
du 25 novembre 1941), question qui soulevait inévitablement celle de la validité de ce décret. L'argumentation de la Cour constitutionnelle a là aussi des accents familiers : La contradiction avec la justice, affirme-t-elle ainsi dans ses attendus, a atteint dans ce décret « un degré si insupportable » (« ein so unerträgliches Maß») que l'on doit considérer qu'il était nul dès le départ ${ }^{(10)}$.

Ces quelques exemples ${ }^{(1)}$ illustrent l'influence de la théorie de la validité de Radbruch sur la justice fédérale; cette influence démontre quant à elle que les juges étaient disposés à recourir à des critères éthiques et moraux et à se référer au droit naturel dans l'application et l'interprétation du droit.

\section{... et infortune d'une formule}

Mais la réception de la pensée de Radbruch ne s'arrête pas là. Ses essais de l'aprèsguerre contiennent en effet une autre thèse qui est tout sauf secondaire: «De fait, le positivisme, avec sa conviction selon laquelle "la loi, c'est la loi" ["Gesetz ist Gesetz"], a rendu la corporation des juristes allemands sans défense (wehrlos) face à des lois de contenu arbitraire et criminel ${ }^{(12)}$. Le coupable ${ }^{(13)}$ de la faillite de la justice sous le Troisième Reich, affirme Radbruch, serait donc le positivisme. L'explication est doublement problématique. Tout d'abord, elle n'est objectivement pas tenable: les juristes s'étaient en effet détournés dès Weimar du positivisme - on se contentera ici de rappeler l'ardeur des juges à revendiquer alors le droit à l'examen de constitutionnalité( ${ }^{(14)}$; en outre, elle conduit à décharger la corporation de sa responsabilité, si ce n'est morale, tout au moins juridique. Comme l'explique Radbruch, pour qu'il y ait responsabilité pénale du juge, il faut qu'il y ait application incorrecte du droit (Rechtsbeugung). Dans le cas de l'application d'une " loi » ou d'un décret national-socialiste non valide, il y a bien objectivement application incorrecte du droit, mais des juges qui étaient si « déformés » (verbildet) par le positivisme dominant qu'ils ne connaissaient pas d'autre droit que celui qui a été posé pouvaient-ils avoir, en appliquant les lois positives, l'intention d'appliquer le droit de façon incorrecte ${ }^{(15)}$ ?

Incontestablement, la mise à l'index du positivisme a contribué à l'accueil favorable des textes de Radbruch par des juristes reconnaissant de trouver là un bouc émissaire tout désigné. La thèse semblait d'autant plus crédible que Radbruch se livrait en fait à une autocritique. Avant 1933, sa philosophie du droit aboutissait en effet, et ce en dépit

10 Jugement du 14 février 1968, BVerfGE, 23, p. 98.

11 Pour d'autres exemples et plus de détails, cf. B. Schumacher, Rezeption und Kritik der Radbruchschen Formel, thèse, université de Göttingen, 1985 et H.-E. DiEcKMAnN, Überpositives Recht als Prüfungsmaßstab im Geltungsbereich des Grundgesetzes? Eine kritische Würdigung der Rezeption der Radbruchschen Formel und des Naturrechtsgedankens in der Rechtsprechung, Berlin, Duncker \& Humblot, 2006.

12 G. Radbruch, «Gesetzliches Unrecht» (note 3), p. 88. Trad. (modifiée) : Michael Walz, p. 312.

13 Ou la « contribution de la science juridique à la culpabilité collective », cf. G. RADBRUCH, «Privatissimum der Rechtspflege " (1947), in: GRGA 14: Staat und Verfassung, 2002, p. 150.

14 Sur cette question, que nous ne pouvons développer ici, voir notamment M. WALther, « Hat der juristische Positivismus die deutschen Juristen im Dritten Reich wehrlos gemacht? Zur Analyse und Kritik der Radbruch-These », in: R. Dreier, W. Sellert (éd.), Recht und Justiz im "Dritten Reich", Francfort-sur-le-Main, Suhrkamp, 1999, p. 323-354.

15 G. Radbruch, «Gesetzliches Unrecht» (note 3), p. 91 sq. 
de son concept de droit non positiviste, à une résolution positiviste du problème de la validité. Faute de pouvoir définir le contenu matériel de la justice en raison de son relativisme, qui ne peut établir la supériorité d'une conception de l'égalité sur une autre, Radbruch s'était vu contraint de se replier sur la sécurité juridique comme seul critère de la validité du droit. Indirectement, l'essai de 1946 donne ainsi une certaine plausibilité au raisonnement de Carl Schmitt, lorsque, en 1958, il présente son texte «Légalité et légitimité » de 1932 comme une tentative pour sauver une constitution de Weimar affaiblie par la doctrine positiviste et illustre celle-ci par une phrase de Radbruch: «Celui qui est en mesure d'imposer le droit prouve par là même qu'il est appelé à poser le droit ${ }^{(16)}$. Quatre ans plus tôt, le pénaliste Hans Welzel utilisait exactement le même passage pour rappeler que c'est formés dans l'esprit de ce positivisme auquel une figure aussi intègre que Radbruch avait donné sa " consécration éthique et philosophique " que les juristes étaient arrivés sous le Troisième Reich. Évidemment, Welzel se garde bien de citer le reste du chapitre en question, où Radbruch expliquait justement que cette phrase ne pouvait pas être le dernier mot de la philosophie du droit ${ }^{(18)}$. Il se garde encore plus de rappeler ses propres prises de position des années $1930^{(19)}$.

Tout le paradoxe est là: en voulant fournir les bases à une reconstruction de l'État de droit, Radbruch a en même temps contribué à escamoter une discussion sur la responsabilité de la corporation et donné à certains juristes compromis sous le nazisme des arguments pour s'intégrer dans le mouvement de renaissance du droit naturel (Naturrechtsrenaissance) de l'après-guerre ${ }^{(20)}$.

Le retentissement de la théorie de Radbruch ne doit par ailleurs pas cacher les résistances auxquelles se heurtent très tôt ses conceptions et les lois ou jugements allant dans leur sens. Dès 1947, l'application de la loi nº 10 de l'autorité de contrôle alliée se voit ainsi contestée au nom du principe de légalité des peines (nulla poena sine lege) qui implique la non rétroactivité de la loi. Ses opposants n’hésitent pas à comparer cette loi

16 "Wer Recht durchzusetzen vermag, beweist damit, daß er Recht zu setzen berufen ist. » G. RADBRUCH, Rechtsphilosophie, in: GRGA 2: Rechtsphilosophie II, 1932, p. 313. Cité par C. Sснмiтt, « Nachbemerkung zum Neudruck von Legalität und Legitimität» (1958), in: ID., Verfassungsrechtliche Aufsätze aus den Jahren 1924-54, Berlin, Duncker \& Humblot, 1985, p. 346.

17 " ethische und philosophische Weihe »: H. Welzel, « Naturrecht und Rechtspositivismus? (1953), in: W. Mainofer, Naturrecht oder Rechtspositivismus, Darmstadt, Wiss. Buchgesellschaft, 1972, p. $322 s q$.

18 G. Radbruch, Rechtsphilosophie (note 16), p. 315: dans la plupart des cas, la conscience individuelle doit juger une violation du droit positif plus grave que le sacrifice de ses propres convictions, mais il peut y avoir des «lois scélérates » (Schandgesetze) auxquelles la conscience refuse l'obéissance. Même si Radbruch refuse alors encore cette possibilité au juge dans l'exercice de sa fonction, on est loin d'un positivisme « pur et dur».

19 Il avait notamment approuvé en 1935 la modification du $\$ 2$ du Code Pénal qui rompait avec le principe de la légalité des peines en rendant passible d'une peine l'auteur non seulement d'un acte réprimé par la loi, mais aussi d'un acte méritant une punition conformément à l'idée fondamentale d'une loi répressive ou au «bon sens populaire» (« nach gesundem Volksempfinden »).

20 I. Müller («Gesetzliches Recht und übergesetzliches Unrecht. Gustav Radbruch und die Kontinuität der deutschen Staatslehre », Leviathan, 7 [1979], p. 323) et M. Walther (" Hat der juristische Positivismus " [note 14], p. 350 sq.) n'hésitent pas à parler d'une falsification de l'histoire du droit à laquelle aurait involontairement contribué Radbruch. 
avec l'évolution du droit sous le nazisme (!), où le principe de légalité avait été lui aussi délaissé au nom d'une justice matérielle supérieure - en l'occurrence celle fondée sur le "bon sens populaire " ${ }^{(21)}$. Radbruch leur réplique en arguant que la loi n'est en fait pas rétroactive si l'on considère que son contenu était déjà en vigueur auparavant, certes pas sous forme de loi positive, mais en tant que droit naturel, supralégal ${ }^{(22)}$. Il souligne également les implications problématiques de l'argumentation des opposants à la loi : un crime comme l'euthanasie des malades mentaux, par exemple, resterait impuni parce qu'il reposait sur un « ordre » de Hitler qui, selon la « constitution » de l'époque, était assimilé à un acte législatif ${ }^{(23)}$. Or, alors que des médecins impliqués dans des actes d'euthanasie sont condamnés sur la base notamment de la théorie de Radbruch, des pénalistes comme Welzel déclenchent une contre-offensive fondée sur la construction de l'erreur de droit (Verbotsirrtum) : les actes des médecins étaient contraires au droit, mais ils ne sont pas coupables car ils croyaient à la légalité de l'ordre nazi ${ }^{(24)}$. On ne peut donc pas vraiment parler d'un «monopole » de la théorie de Radbruch quant à l'appréhension de la période national-socialiste par les juristes.

En revanche - nouveau paradoxe -, la théorie de Radbruch a aussi été utilisée hors de son champ d'application. Contrairement à d'autres tenants de la Naturrechtsrenaissance, celui-ci a toujours insisté sur les limites et les dangers du concept de " droit supralégal » et de l’appel au droit naturel pour la sécurité juridique. La nécessité de restaurer non seulement la justice, mais également la sécurité juridique, est ainsi un leitmotiv de ses textes des années 1945-48, de même que l'idée que le recours au concept de « droit supralégal » doit rester limité à des cas exceptionnels, à la « situation totalement unique » (« den völlig singulären Verhältnissen ») du régime nazi ${ }^{(25)}$. Or, la première référence à Radbruch dans un jugement du Bundesverfassungsgericht se situe sur un tout autre plan, puisqu'il y est question de la possibilité de vérifier la conformité d'une norme constitutionnelle, en l'occurrence l'article 117 de la Loi fondamentale, avec des normes suprapositives. La leçon du national-socialisme, estiment les juges, est que dans certains cas extrêmes, qu'ils ne voient pas l'utilité de préciser, il faut pouvoir placer la justice matérielle au-dessus de la sécurité juridique, le caractère exceptionnel de ces situations ayant été très bien formulé par Radbruch: c'est là qu'intervient la

21 Voir notamment H. von Hodenberg, «Zur Anwendung des Kontrollratsgesetzes Nr. 10 durch deutsche Gerichte », SJZ, 2 (1947), p. 113-124. Voir aussi J. Perels, " Die Restauration der Rechtslehre » (note 7), p. 44 sq.; C. LAAGE, « Die Auseinandersetzung um den Begriff des gesetzlichen Unrechts nach 1945 », Kritische Justiz, 22 (1989), p. 427 sqq.

22 G. Radbruch, «Zur Diskussion über die Verbrechen gegen die Menschlichkeit », SJZ, 2 (1947), p. 135: le peuple allemand avait-il à ce point perdu l'esprit qu'il ne lui fût jamais venu à l'idée, par exemple dans le cas de l'euthanasie des malades mentaux, qu'il s'agissait malgré «l'ordre » du Führer d'injustice légale?

23 Ibid., p. 134.

24 En 1947, l'Oberlandesgericht de Francfort avait condamné des médecins en argumentant que l'ordre de Hitler n'était pas une « loi " même du point de vue des nazis, puisqu'il avait été gardé secret, mais également selon des critères de droit naturel, la cour se référant sur ce point à la formule Radbruch. Cf. SJZ, 2 (1947) (avec un commentaire de Radbruch), p. 632-636. Pour Welzel, voir J. Perels, « Die Restauration der Rechtslehre» (note 7), p. 46 sq.

25 G. Radbruch, "Zur Diskussion » (note 22), p. 136. Voir aussi " Privatissimum der Rechtspflege » (note 13), p. 152 sq.; "Gesetzliches Unrecht» (note 3), p. 90; "Die Erneuerung des Rechts » (note 2), p. 107; Vorschule der Rechtsphilosophie, in: GRGA 3, p. 154. 
citation de la formule ${ }^{(26)}$. La référence est donc utilisée pour légitimer la compétence de la Cour à rejeter des normes constitutionnelles sur la base de principes suprapositifs auxquels serait lié le pouvoir constituant.

On peut affirmer sans trop de risques que Radbruch n'aurait pas apprécié cette application " extensive " de sa formule, et ce d'autant plus que la Loi fondamentale avait entre-temps intégré dans le droit positif certains principes qui lui permettaient de définir le droit supralégal (protection de la dignité humaine, etc.). De même, le pionnier de la réforme du droit pénal qu'il était ne se serait vraisemblablement pas retrouvé dans la tendance qui se manifeste dans certaines décisions judiciaires des années 1950, comme ce jugement de 1954 dans lequel le Bundesgerichtshof estime que des relations sexuelles avant le mariage relèvent du délit d'attentat aux mœurs (Unzucht) en se référant aux « normes de la loi morale» ("Normen des Sittengesetzes») - exemple parmi d'autres de la façon dont le droit naturel ou des principes moraux supérieurs sont invoqués pour défendre des valeurs conservatrices ${ }^{(27)}$. Mais on peut justement considérer que le discours de Radbruch sur le droit supralégal et le droit naturel, où se mélangent références bibliques, renvois au droit naturel catholique et au droit naturel rationnel des Lumières, a contribué sans le vouloir à légitimer un appel indifférencié au droit naturel qui pouvait pourtant avoir des arrière-plans éthiques et idéologiques fort différents.

\section{La refondation de la démocratie: Radbruch et le poids de l'héritage weimarien}

Outre le principe de l'État de droit, un autre principe - un autre « fondement normatif »- est protégé par la « clause d'éternité » de la Loi fondamentale: la démocratie. Là encore, la figure de Radbruch pouvait présenter un certain intérêt dans le cadre de cette refondation démocratique. Non seulement il avait fait partie des - rares - juristes à défendre Weimar, mais il avait en outre développé une pensée pluraliste de la démocratie qui tranchait alors singulièrement avec le discours dominant. Cette conception transparaît notamment dans les textes où il analyse et défend le rôle des partis politiques. Alors que les partis sont à l'époque massivement dénigrés et associés à l'idée de désintégration de la communauté, Radbruch milite pour leur intégration dans la constitution et défend la thèse que la démocratie moderne ne peut être qu'un État de partis (Parteienstaat) ${ }^{(28)}$. Il procède même à une double légitimation de l'État de partis, à partir de la réalité sociologique d'une part - dans une démocratie de masse, l'exercice de la souveraineté populaire ne peut être envisagé sans les partis politiques - et à partir de son relativisme philosophique d'autre part: étant donné qu'il est impossible de démontrer la supériorité d'une vision du monde sur une autre, Radbruch a toujours considéré le combat des partis politiques comme une évidence. Son discours avait ainsi le mérité de disqualifier des idéologies comme celle de l'homogénéité de la volonté du peuple au sein de la Volksgemeinschaft ou de l'État neutre au-dessus des partis. Il véhiculait en outre une éthique politique fondée sur la tolérance et le compromis qui est à la base de la démocratie.

26 Jugement du 18 décembre 1953, BVerfGE, 3, p. 224 sqq. (232).

27 BGHSt, 6, p. 52 (« Kuppeleibeschluß»).

28 Cf. G. Radbruch, «Parteienstaat und Volksgemeinschaft » (1929), in: GRGA 12: Politische Schriften der Weimarer Zeit I, 1992, p. 94-99 et « Die politischen Parteien im System des deutschen Verfassungsrechts » (1930), in: G. ANschütz (éd.), Handbuch des deutschen Staatsrechts I, Tübingen, Mohr, 1930, p. $285-294$. 


\section{Une réception limitée}

Néanmoins, force est de constater que les références aux conceptions de la démocratie de Radbruch restent limitées après 1945. L'article 21 de la Loi fondamentale va certes dans son sens en reconnaissant le rôle des partis dans le processus de formation de la volonté politique. Il ne faut toutefois pas surestimer l'influence des théoriciens weimariens sur les décisions du Parlamentarischer Rat ${ }^{(29)}$. Les décisions de la Cour constitutionnelle, en revanche, contiennent des références à Radbruch. En 1952, elle définit par exemple comme suit le rôle des partis dans la démocratie: "Ils sont, comme Radbruch [Handbuch des deutschen Staatsrechts, vol. 1, 1930, p. 288] l'a déjà souligné avec raison, les "ultimes organes de création de tous les autres organes [...] sans l'intervention desquels la masse amorphe du peuple serait totalement incapable de faire émerger les organes du pouvoir étatique". Aujourd'hui, toute démocratie est nécessairement un État de partis ${ }^{(30)}$. On trouve une autre référence intéressante dans le jugement de 1956 sur l'interdiction du parti communiste (KPD): dans une démocratie, tient à rappeler la Cour constitutionnelle, aucun parti politique ne peut prétendre que seuls ses buts politiques sont justes; " dans le domaine des convictions politiques fondamentales, il n'existe pas de vérité démontrable et irréfutable " (cf. Radbruch, HdbDStR, vol. 1, p. 289) ${ }^{(31)}$.

Les textes « weimariens » de Radbruch ne sont donc pas ignorés, mais l'interprétation de ces références doit être nuancée: tout d'abord, elles sont rares; plus encore, si la décision de 1952 cite Radbruch, elle porte surtout la marque de Gerhard Leibholz, alors membre de la deuxième chambre de la Cour constitutionnelle, et de sa Parteienstaatstheorie. Elle reprend même pratiquement mot pour mot des phrases de ses écrits ${ }^{(32)}$. Or on ne peut pas dire que les références à Radbruch soient nombreuses chez Leibholz; elles sont en outre, excepté celles ayant trait à sa critique de l'idéologie de l'État autoritaire $^{(33)}$, négatives ${ }^{(34)}$ - Leibholz critique notamment la tentative de concilier l'idée du Parteienstaat et l'article 21 de la Constitution de Weimar qui précisait, comme la Loi fondamentale d'ailleurs, que les députés sont soumis à leur seule conscience et ne sont

29 Voir V. Отто, Das Staatsverständnis des Parlamentarischen Rates. Ein Beitrag zur Entstehungsgeschichte des Grundgesetzes für die Bundesrepublik Deutschland, Bonn/Bad Godesberg, Droste, 1971, p. 173: l'article 21 venait moins sanctionner le résultat d'une réflexion théorique qu'un état de fait, et beaucoup de membres du Parlamentarischer Rat étaient encore très réticents envers le phénomène des partis; M. MorLock, "Entdeckung und Theorie des Parteienstaats ", in: Christoph Gusy (éd.), Weimars lange Schatten. "Weimar” als Argument nach 1945, Baden-Baden, Nomos, 2003, p. 243.

30 Jugement du 5 avril 1952, BVerfGE, 1, p. 223: « die “letzten Kreationsorgane aller anderen Organe... ohne deren Zwischenschaltung die amorphe Volksmasse gar nicht imstande wäre, die Organe der Staatsgewalt aus sich zu entlassen”. Heute ist jede Demokratie zwangsläufig ein Parteienstaat. »

31 Jugement du 17 août 1956, BVerfGE, 5, p. 224: « daß es im Bereich der politischen Grundanschauungen eine beweisbare und unwiderlegbare Richtigkeit nicht gibt ». La citation est extraite du même article de Radbruch.

32 Par exemple la phrase: "Sie erscheinen geradezu als das Sprachrohr, dessen sich das mündig gewordene Volk bedient, um sich artikuliert äußern und politische Entscheidungen fällen zu können », que l'on trouve déjà à quelques détails près dans un texte de 1950, "Volk und Partei im neuen deutschen Verfassungsrecht » (G. LeibHolz, Strukturprobleme der Demokratie, Karlsruhe, Müller, 1967, p. 76). 
liés à aucune directive. En dépit de certains points communs, la Parteienstaatstheorie de Leibholz, qui avait été l'élève de Carl Schmitt, ne revendique ainsi aucune filiation avec celle de Radbruch ${ }^{(35)}$.

\section{Le relativisme face à la "démocratie militante »}

Plusieurs raisons permettent d'expliquer cette réception limitée. Des raisons d'ordre méthodologique et liées à sa spécialité scientifique, tout d'abord: Radbruch n'était pas un constitutionnaliste, et sa théorie de l'État de partis en est restée à l'état d'esquisse. À cela s'ajoutent les circonstances, qui ont fait que Radbruch n'a pas pu commenter la Loi fondamentale, tandis qu'un juriste comme Leibholz accédait à une position influente. L'impact de la formule Radbruch n'est pas non plus à sous-estimer : elle a en effet focalisé l'attention sur sa philosophie du droit tout en invitant à une prise de distance par rapport à ses écrits weimariens.

Mais l'explication principale réside à notre sens ailleurs - dans un aspect de la pensée de Radbruch qui s'avère rédhibitoire: son relativisme. Car s'il y a une idée qui fait consensus durant cette période de l'après-guerre ${ }^{(36)}$, c'est bien celle que la "neutralité » absolue de la Constitution de Weimar et son incapacité à se défendre contre ses ennemis ont conduit à son "suicide ». Le relativisme apparaît comme le fondement philosophique d'une conception formaliste, et non normative, de la démocratie qui conduit à mettre la constitution entièrement à la disposition de la majorité. Or Radbruch était, avec Hans Kelsen, considéré comme le principal représentant de ce relativisme. Sous Weimar, il avait affirmé comme le juriste autrichien que le relativisme était le présupposé de l'idée démocratique, expliquant que l'esprit de la constitution se résumait aux principes formels de décision majoritaire et de tolérance ${ }^{(37)}$. En 1934, il avait toutefois, mais sans pour autant renoncer au relativisme, infléchi sa conception en affirmant que l'État démocratique devait certes tolérer toutes les opinions, mais pas celle qui se prétend absolue et ne respecte pas les règles du jeu démocratique ${ }^{(38)}$.

Il n'est donc finalement pas étonnant que les références aux écrits weimariens de Radbruch restent limitées, voire absentes dans des discours qui stigmatisent la neutralité de la démocratie weimarienne et qui font de cette critique un élément de légitimation de la République fédérale («Bonn n'est pas Weimar»). Cela vaut pour les débats au Parlamentarischer Rat $^{(39)}$; cela vaut pour les textes de Leibholz, qui juge sans indulgence

35 Elle repose de fait sur des prémisses théoriques différentes, Leibholz raisonnant toujours en termes d'opposition entre démocratie représentative et identitaire et considérant le Parteienstaat comme une forme de démocratie plébiscitaire dans laquelle la volonté générale se constitue par identification avec la volonté du parti majoritaire. Radbruch, en revanche, avait congédié très tôt toute idée d'« homogénéité » ou d'«identité » entre gouvernants et gouvernés et sa conception apparaît ainsi plus résolument pluraliste que celle de Leibholz.

36 Et apparue dès les années $1930 \mathrm{chez}$ les émigrés, cf. Karl Löwenstein, " Militant democracy and Fundamental Rights », American political science review, 31 (1937), p. 417-433 et 638-658.

37 Cf. G. RAdbruch, « Parteienstaat und Volksgemeinschaft » (note 28), p. 97, 99.

38 «Relativismus ist die allgemeine Toleranz - nur nicht Toleranz gegenüber der Intoleranz. » G. RADBRUCH, «Der Relativismus in der Rechtsphilosophie », in: GRGA 3, 1934, p. 21.

39 Voir les critiques des députés aussi bien issus du SPD (en particulier Carlo Schmid) que de la CDU et CSU lors des premières séances plénières du Parlamentarischer Rat les 8 et 9 novembre 1948 (voir Der Parlamentarische Rat 1948-49. Akten und Protokolle, vol. 9: Plenum, Boppart am Rhein, Boldt, 1997). 
la conception " erronée » de la liberté fondée sur un "nihilisme et un relativisme sans aucune substance » ("substanzloser Relativismus und Nihilismus ») qui était à la base de la Constitution de Weimar ${ }^{(40)}$; cela vaut enfin pour les décisions de la Cour constitutionnelle, qui pour justifier l'interdiction du KPD s'appuie sur le principe de la "démocratie combative » ou " militante " ( streitbare Demokratie »), lui-même légitimé par l'expérience de Weimar - celle d'une constitution sans défense victime de son indifférence politique ${ }^{(41)}$. La critique de Kurt Sontheimer dans son ouvrage sur la pensée antidémocratique sous Weimar, paru en 1962, va dans le même sens. Selon lui, les relativistes, soit Kelsen et Radbruch, ont fondamentalement mal compris la démocratie en y voyant une forme de gouvernement sans aucun contenu normatif et tolérant toutes les convictions. Si la conception relativiste est "inoffensive " tant que règne un consensus général sur les fondements de l'État, elle a des conséquences fatales dès que ce consensus se disloque et conduit à la capitulation de la démocratie ${ }^{(42)}$.

Nous ne pouvons nous attarder sur tout ce que ces condamnations impliquent d'amalgames - entre relativisme et nihilisme ou indifférence éthique, amalgame que vient pourtant contredire l'engagement d'un Radbruch ou d'un Kelsen -, de simplifications et d'ambivalences - se référer, comme le fait Sontheimer et comme l'ont fait beaucoup d'autres, à Rudolf Smend, qui n'avait pas vraiment été un défenseur acharné de Weimar et dont la théorie de l'intégration était très éloignée d'un modèle rationnel de construction de la volonté politique, ne va pas sans poser problème. Il faut en revanche souligner un paradoxe qui n'est pas sans ironie. Car c'est bien dans le même jugement de 1956 où les membres du Bundesverfassungsgericht critiquent la neutralité de la Constitution de Weimar qu'ils citent une phrase de Radbruch (cf. note 31) qui n'est autre qu'une définition du... relativisme. Certes, les juges en tirent la conclusion, comme du reste Radbruch en 1934, que la démocratie doit être intolérante envers un parti prétendant détenir la seule vérité politique, ce qui correspond au principe de la démocratie combative. La référence démontre cependant qu'il existe bien des convergences entre la pensée de Radbruch et les principes, ou « l'esprit », pour reprendre un terme qu'il affectionnait, de la nouvelle démocratie, même si elles ne sont pas mises en avant.

Le contraste et les ambivalences qui caractérisent la réception de la pensée de Radbruch dans les deux domaines analysés ${ }^{(43)}$ apparaissent en définitive - ultime paradoxe - tout à fait cohérents. Mais cette cohérence se situe moins sur le plan de la théorie juridique qu'au niveau des facteurs idéologiques et politiques et du rapport au passé qui entrent en jeu dans la refondation de l'État dans l'Allemagne de l'après-guerre. C’est la volonté de tirer les leçons du nazisme qui a amené Radbruch à modifier sa théorie de la validité du droit et à rejeter le positivisme. C'est la volonté d'«évacuer »

40 G. Leibholz, «Freiheitliche demokratische Ordnung und das Bonner Grundgesetz » (1951), in: ID., Strukturprobleme der Demokratie (note 32), p. 139.

41 Voir BVerfGE, 5, p. 138.

42 Cf. K. Sontheimer, Antidemokratisches Denken in der Weimarer Republik (1962), Munich, DTV, 1994 , p. 85, 181 sq.

43 On aurait aussi pu s’intéresser à la réception de sa théorie du droit social ou à celle de ses réflexions sur l'éducation civique dans le cadre de la démocratie, question aussi débattue dans la jeune République fédérale. Mais cela aurait dépassé le cadre de cette contribution. 
la question de la responsabilité des juges sous le Troisième Reich qui a par ailleurs contribué à l'accueil favorable de ses écrits. En voulant marquer la rupture avec la période nazie, Radbruch a ainsi paradoxalement favorisé une certaine continuité au niveau de la corporation des juristes et est devenu l'emblème, voire la caution d'une renaissance du droit naturel dont certains aspects ne correspondaient pas à sa pensée. C'est enfin toujours la volonté de tirer des leçons, mais cette fois de l'échec de Weimar, qui explique en partie la faible réception de ses réflexions antérieures sur la démocratie. Le rôle joué par la pensée de Radbruch nous renvoie finalement aux rapports ambivalents qu'entretient la jeune République fédérale avec Weimar, mais aussi avec la période national-socialiste.

\section{Zusammenfassung}

Auf welchen Grundlagen konnte ein demokratischer Rechtsstaat auf den Trümmern des totalitären Regimes wiederaufgebaut werden? Nach 1945 konnte das Denken des Juristen Gustav Radbruch Anknüpfungspunkte dafür bieten. Einerseits wirkte Radbruch in der Tat am Wiederaufbau des Rechtsstaats mit, indem er das theoretische Rüstzeug für die juristische Aufarbeitung der in der NS-Zeit begangenen Verbrechen lieferte; andererseits hat aber seine These, der Positivismus sei für das Versagen der Justiz im Dritten Reich verantwortlich, auch zur Entlastung des Juristenstandes und zu einer ambivalenten Naturrechtsrenaissance beigetragen. Außerdem wurde Radbruchs demokratisches Denken eher wenig rezipiert, obwohl er zu einem der wenigen Weimarer Juristen gehörte, die überzeugte „Demokraten“ waren. Letztendlich weisen die Paradoxe der Radbruchschen Rezeption auf die Ambivalenzen hin, die das Verhältnis der jungen Bundesrepublik zum Erbe der Weimarer Republik und des Dritten Reiches kennzeichnen.

\section{Résumé}

Sur quelles bases refonder un État de droit démocratique sur les ruines du régime totalitaire? Après 1945, la pensée d'un juriste comme Gustav Radbruch pouvait apparaître comme un possible point d'ancrage. Mais si elle a en effet contribué à la reconstruction de l'État de droit en fournissant à la justice les instruments théoriques pour traiter les crimes commis sous le nazisme, sa thèse de la responsabilité du positivisme dans la faillite de la justice sous le Troisième Reich a aussi conduit à disculper le corps judiciaire et favorisé une renaissance du droit naturel aux implications ambivalentes. Par ailleurs, alors que Radbruch était un des rares juristes "démocrates " de Weimar, sa théorie de la démocratie n'a en revanche connu qu'une réception limitée. Les paradoxes repérés dans la réception de la pensée de Radbruch témoignent en fait finalement de ceux de la jeune République fédérale elle-même face à l'héritage de Weimar et du Troisième Reich. 